\title{
The PRIMA Astrometric Planet Search: Goals and Prospects
}

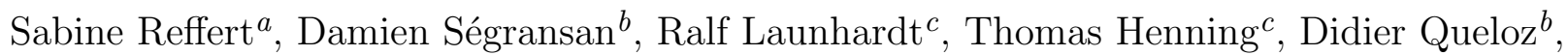 \\ Andreas Quirrenbach ${ }^{a}$, Francesco Pepe ${ }^{b}$, Johny Setiawan $^{c}$, Patrick Weise ${ }^{c}$ \\ aZAH-Landessternwarte, Königstuhl 12, 69117 Heidelberg, Germany \\ ${ }^{b}$ Observatoire de Genève, 51, chemin des Maillettes, CH-1290 Sauverny, Switzerland \\ ${ }^{c}$ Max-Planck-Institut für Astronomie, Königstuhl 17, 69117 Heidelberg, Germany
}

\begin{abstract}
ESO's PRIMA (Phase-Referenced Imaging and Micro-arcsecond Astrometry) facility at the VLT Interferometer on Cerro Paranal in Chile is expected to be fully operational in only a couple of years from now. With PRIMA/VLTI, it will then be possible to perform relative astrometry with an accuracy of the order of $10 \mu$ as over angles of about $10^{\prime \prime}$.

The main science driver for this astrometric capability is the detection and characterization of extrasolar planets, including (1) the observation of known radial velocity planets and planetary systems to fully constrain their orbital geometry and accurately determine the mass of the planet(s), (2) a search for extrasolar planets around stars which are less suitable for the radial velocity method (for example young and active stars as well as early type stars), and (3) a systematic search around the most nearby stars to detect low mass planets (Uranus or Neptune masses).

Preparatory observations of possible target stars, with the aim of identifying nearby suitable astrometric reference stars, have already started with SOFI at the NTT and are described. Furthermore, we compare the goals and prospects of the PRIMA Astrometric Planet Search to other projects aiming at detecting planets astrometrically, mostly from space.
\end{abstract}

Keywords: PRIMA, VLTI, Interferometry, Extrasolar Planets, Astrometry, Reference Star

\section{INTRODUCTION}

ESO's PRIMA (Phase-Referenced Imaging and Micro-arcsecond Astrometry) instrument at the VLTI on Cerro Paranal in Chile is expected to be available for testing by mid 2007 and fully operational by 2008. It consists of four subsystems (star separator, differential delay lines, laser metrology and fringe sensor units), which together will enable differential astrometric measurements over small angles on the sky $\left(\leq 30^{\prime \prime}\right)$ with an accuracy approaching $10 \mu$ as (see e.g. Quirrenbach et al. ${ }^{1,2}$ or Pepe et al. ${ }^{3}$ in this volume). The primary science driver for microarcsecond astrometry is a search for extrasolar planets.

The selection of suitable target and reference stars is by no means an easy task, since all stars have to fulfill tight requirements on astrometric stability. Furthermore, the target stars have to be bright enough for fringetracking in the K-band, and suitable reference stars have to be found within the isoplanatic patch of the target stars. Additionally, standard star pairs in which the separation of the companions can be predicted with high precision in order to build up confidence in our measurements are needed. More details for the selection process of each of those groups of stars can be found in Frink et al. ${ }^{4}$ and Reffert et al. ${ }^{5}$; below, we briefly review the properties of the selected candidate stars and describe the first results of our preparatory observations to make the final selection of the stars which we plan to observe in the guaranteed time granted to our consortium.

E-mail: sreffert@lsw.uni-heidelberg.de 


\section{TARGET STARS, REFERENCE STARS AND STANDARD STAR PAIRS}

\subsection{Target Stars}

Three different groups of target stars are currently considered for our astrometric planet search program:

\section{- Stars with known radial velocity planets}

For stars with known radial velocity planets PRIMA measurements will be able to constrain the inclination and therefore provide an unambiguous measurement of the companion mass, which is not possible with radial velocity measurements alone because of the $\sin i$ uncertainty. The ascending node, which is also not accessible to radial velocities, will also be measured but is of less scientific interest since it only determines the orientation of the projected orbit on the sky. (Note, however, that the orientation of the orbit becomes an interesting quantity if the orientation of the stellar rotation axis can also be measured interferometrically - e.g. Quirrenbach ${ }^{6}$.) In stars with several planetary companions, the inclinations of the various orbits can be measured individually and it can be investigated whether they are co-planar or not.

A second goal of observing stars with known radial velocity planets is a search for additional companions in those systems; it has been shown that the likelihood of finding another planetary companion in a system with already detected radial velocity planets is higher than finding planetary companions in systems with no previously known planets: from their survey of 1330 GKM main-sequence stars Marcy et al. ${ }^{7}$ find that at least $6.6 \%$ (88 stars) harbor extrasolar planets, but that at least $14 \%$ of those detected systems harbor at least another planet (not taking detection biases in the RV method into account, so the real numbers are likely higher). This shows that we might find additional planets around those stars with known radial velocity planets, in particular those where the outer planets are the less massive ones (the systems known to date usually have their more massive planets in the longer orbits, which might be due to the fact that the radial velocities are more sensitive to short period orbits, while the opposite is true for the astrometric method).

\section{- Most nearby stars}

The most nearby stars are the ones which are in principle best suited for an astrometric planet search since the astrometric signal scales inversely with distance. By choosing the most nearby stars we are thus sensitive to low-mass planets which we would miss if the stars were located at larger distances. This is the reason why many of the other pointed astrometric planet searches like e.g. SIM Planetquest will probably focus on the same targets. We have provisionally selected candidate target stars from a preliminary version of the Nearby Stars Catalog (CNS4, Jahreiß, private communication); the final cut in distance will depend on how many of these candidate targets are bright enough in the K-band for fringe-tracking (the very low-mass stars might be too faint even if they are nearby), and on how many of those candidates have suitable reference stars in our SOFI near-IR images.

Around those nearby stars, we will have to execute a 'blind search' for planets. Thus, this makes only sense if the sample is large enough so that one has a high probability of finding any planets. Marcy et al. ${ }^{7}$ extrapolate that about $12 \%$ of stars harbor planets, of which most are probably detectable astrometrically around nearby stars. Thus, the number of targets in this group should be at least of order fifty or so in order to produce interesting scientific results.

\section{- Young stars}

So far, radial velocity planet searches have usually avoided observing young and active stars because of the additional complications posed by interpreting the radial velocity, which might be influenced by the stars' activity. For astrometry, this is less of a problem, although photocenter shifts caused by starspots could also impact the astrometric precision (Launhardt et al., in preparation). However, since the discovery of planets around young stars is of particular scientific interest because of its potential links to the formation scenario of stars and planets, we will include young stars up to distances of about $140 \mathrm{pc}$ (which includes some of the most prominent nearby star-forming regions and associations) in our target list. 


\subsection{Reference Stars}

In dual-star interferometry the position of the target star is measured relative to a nearby astrometric reference star. Since in the narrow-angle regime the astrometric error scales linearly with the separation between target and reference star $^{8}$, the reference star should be located close to the scientific target. For typical atmospheric conditions on Paranal, von der Lühe, Quirrenbach \& Koehler ${ }^{9}$ estimate that the astrometric reference star should not be located more than $10^{\prime \prime}$ away from the target star if the highest accuracy of about $10 \mu$ as is required. Furthermore, reference stars should have a brightness of at least about 16 mag in $K$, and possibly much brighter to reduce the photon noise error which can for fainter stars dominate over the astrometric error due to atmospheric motions.

The identification of suitable reference stars closer than about $30^{\prime \prime}$ around the stars selected from the three various target groups above is the prime objective of our preparatory observing program (NIR imaging) with SOFI at the NTT, described in more detail below.

\subsection{Standard Star Pairs}

The selection of suitable astrometric standard stars for validation of other PRIMA measurements is also not straightforward. There is no single system on the sky for which the separation at any given time can be predicted with microarcsecond accuracy and which could be used as standard star pair for PRIMA. (Rather accurate astrometric measurements of the visual binary $\delta$ Equ were presented by Muterspaugh et al. ${ }^{10}$ recently, with the median of the minor axis of the uncertainty ellipse only $26 \mu$ as and $465 \mu$ as for the major axis, but the separation of the components in this system is only $0.25^{\prime \prime}$, too close for observations with PRIMA.) However, for many systems a reasonable rate of change of the separation can be predicted, especially after the first PRIMA measurements are available, so that - if a few pairs are observed every night with PRIMA - good standard star pairs should be available after commissioning.

Visual binary stars are the best choices as astrometric standard stars, since one gets rid of the effects of relative parallax and proper motion, at least to a large extent. If the standard star pair was not a physical pair and consequently displayed relative parallax and proper motion, these parameters would have to be known with microarcsecond precision before the pair could be considered a good astrometric standard for PRIMA. However, since there is again no system for which these astrometric parameters are known with microarcsecond precision, they would first have to be measured with PRIMA, which takes at least 1.5 years to disentangle the effects of proper motion and parallax. In order to get around these difficulties, it seems to be advantageous to use only physical pairs as astrometric standard star candidates.

There are different approaches to select these physical pairs: one could either pick systems for which an orbital solution is available, so that one can predict the separation at a given time as well as its rate of change, or one could select systems with indications for very long periods, so that no orbital motion (or at least nothing except for a linear term) is detectable with PRIMA.

It turned out that none of the visual binary star pairs with accurately known orbital solutions and observable with PRIMA has predicted separations with an accuracy of better than about 8 mas, not anywhere near the PRIMA accuracy. The errors in the predicted rates of change of the separations are also rather large, so that we instead decided to use the stars with the smallest upper limits on the predicted rate of change of the separations. In the Sixth Catalog of Orbits and Visual Binary Stars by Hartkopf \& Mason ${ }^{11}$ we could find 20 pairs observable with PRIMA and having upper limits on their predicted rate of change in the separation of less than $50 \mu \mathrm{as} /$ day. This is still not quite as good as the PRIMA accuracy of $10 \mu \mathrm{as}$, but we estimate that we can derive more accurate rates of change once we have measured those stars extensively during commissioning, so that they can be used as standard star pairs afterwards for the regular observing.

Systems with no detected orbital motion were also investigated for their suitability as PRIMA standard star pairs. In addition to the almost 2000 systems in Hipparcos with F-type solutions in the Component Solutions of Double and Multiple Stars Annex to the Hipparcos Catalogue, where both components have been solved with identical parallax and proper motions, we looked for long period visual pairs in the Washington Double Star Catalog (Mason, Wycoff \& Hartkopf 2004) and found 19 pairs observable with PRIMA for which no orbital motion has been detected over at least 20 years. If we assume that the separation could be measured with an 


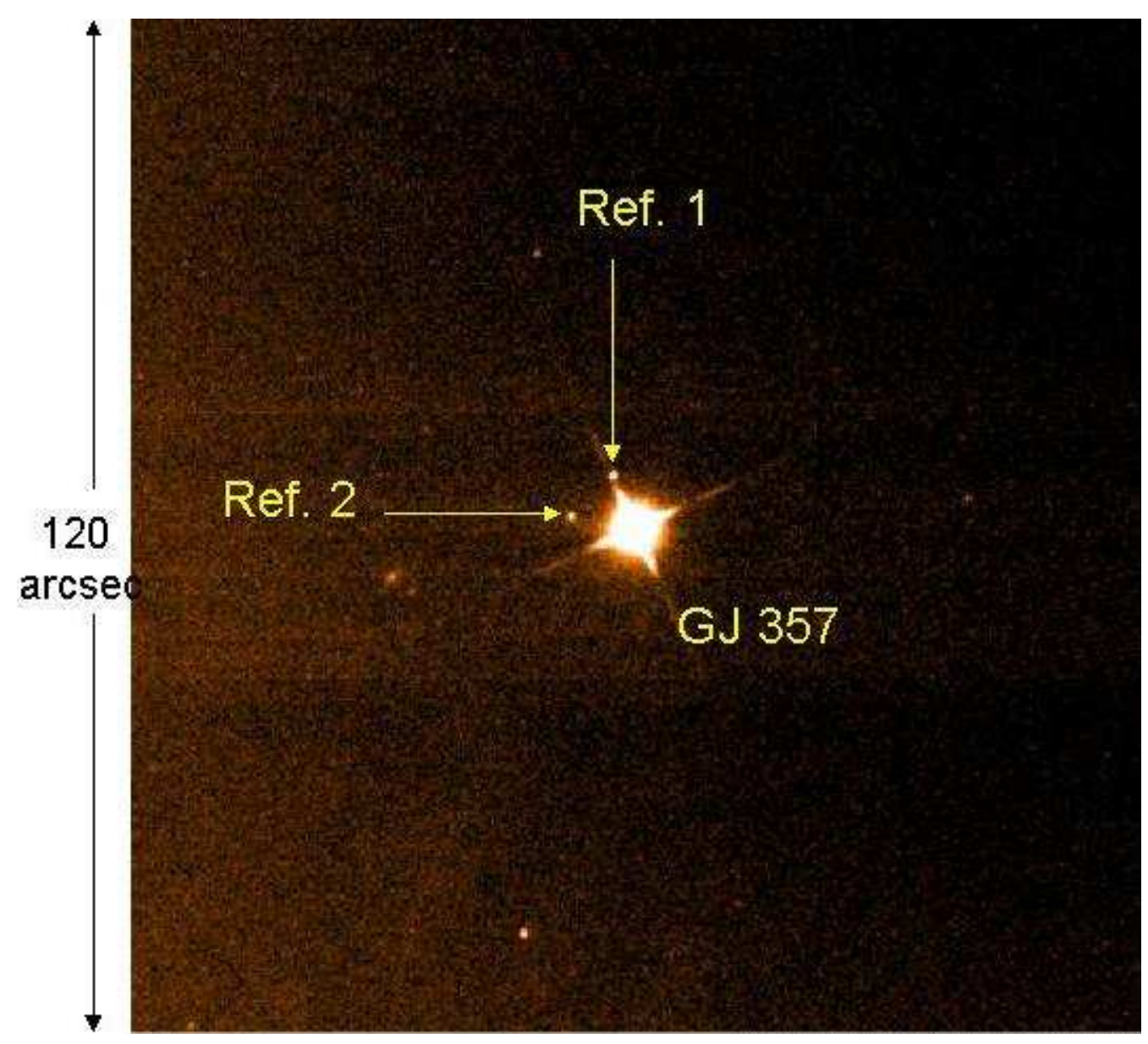

Figure 1. Example of our SOFI images around the nearby star GJ 357. The extent of the image is $2^{\prime \prime}$ on each side. Two possible reference stars close to the star are indicated by arrows and labeled 'Ref. 1' and 'Ref. 2'.

accuracy of $0.1^{\prime \prime}$, and that it did not change over 20 years, this places an upper limit of $14 \mu$ as/day on the rate of change of the separations, comparable or even slightly better than derived for the pairs with the most accurate orbital solutions.

However, systems with no detected orbital motion always hold the risk of not being physical pairs, which implies that they would have to be solved for individual parallaxes and proper motions and are thus less well suited as astrometric standard star pairs, so both types of standard star pairs discussed above will probably be used.

\section{PREPARATORY OBSERVING PROGRAM}

In order to identify suitable reference stars around our targets, we started in 2004 to observe stars of the three target star groups discussed in Section 2. So far, we have observed and reduced 104 fields, observations of 114 more fields have been taken but not fully reduced yet, and more observing time is coming up in 2006 . All observations were carried out with the near infrared imager SOFI at the ESO 3.6 m NTT telescope on La Silla in Chile through $J$ and $K$ filters. Stars can be detected down to about 16 mag in $K$ on these images, exactly as needed for our purposes. Depending on the brightness of the target star, the sensitivity in the inner few arcseconds is much reduced, and usually only true physical companions of similar brightness as the target star can be detected. This is not a severe limitation, considering that it is very unlikely to find an unrelated field 


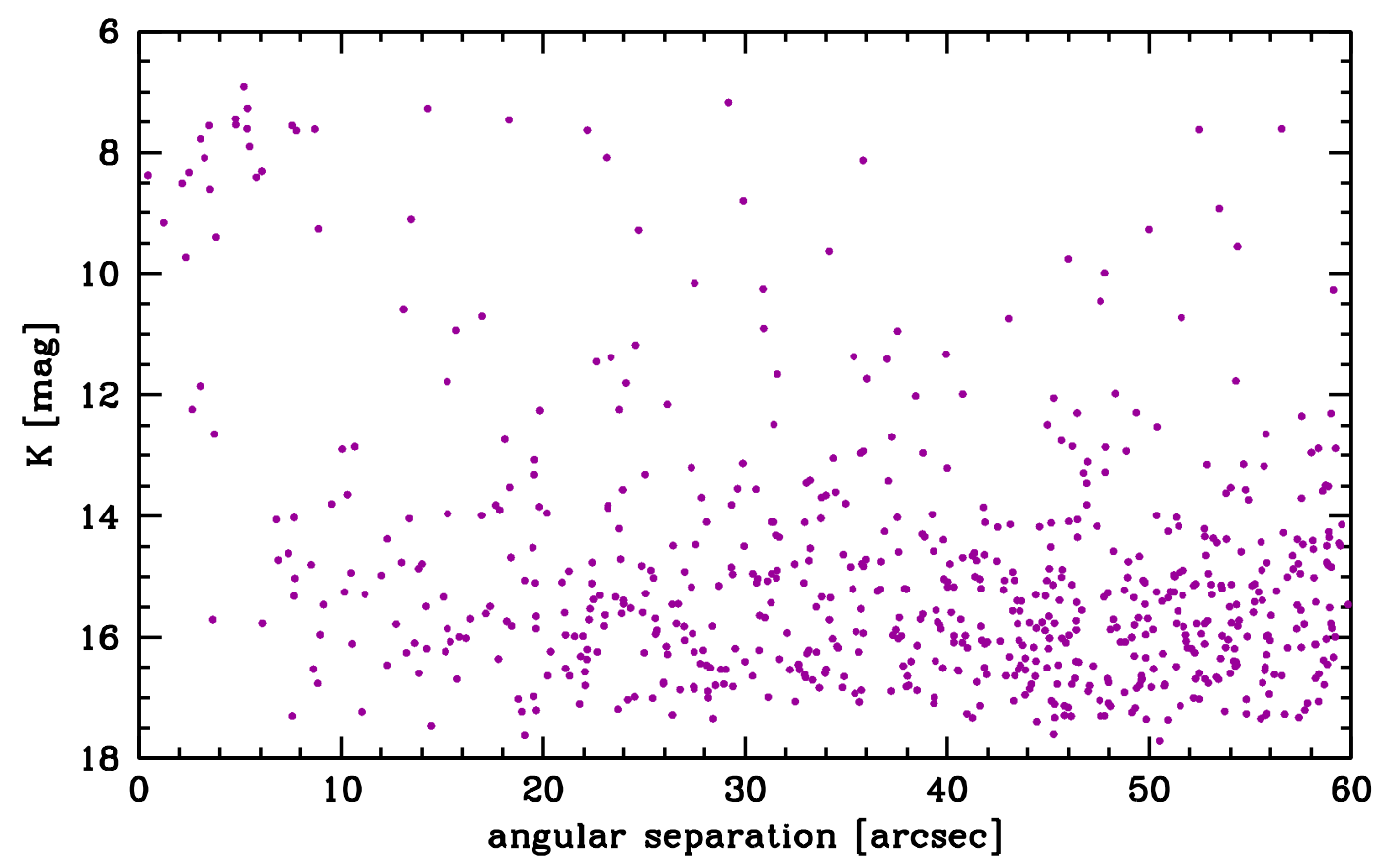

Figure 2. All 801 companions which have been detected in the K-band within $60^{\prime \prime}$ around the target star in 100 fields, including binary companions of comparable brightness as the target star. Within about $4^{\prime \prime}$ of a bright target it is not possible to detect fainter companions in our SOFI images.

star within the given magnitude limit in such a small region; see also the distributions of angular separations in Figs. 2 and 4.

An example of one of our images with two possible reference stars within $10^{\prime \prime}$ of the target is shown in Fig. 1. In Fig. 2 we show the distribution of angular separations and $K$ magnitudes of all 801 stars detected in the $K$ band in 100 fields; in 4 fields, no stars were detected within about 80" of the target. The stars at bright $K$ magnitudes and small angular separations in the upper left corner of the plot are (known) physical companions, while the other stars in the lower right corner of the plot are probably mostly unrelated field stars. Judging from the distribution of $J-K$ colors shown in Fig. 3 the majority of the identified stars is of spectral types G and K. Probably most of them are giants, but there might also be some dwarfs among them. The spectral types at the top scale of the plot are based on the intrinsic $J-K$ colors for giants as given by Bessell \& Brett $^{12}$; intrinsic $J-K$ colors would be approximately $0.1-0.15 \mathrm{mag}$ bluer for main-sequence stars of the same spectral type in that range.

In Fig. 3, only stars which were detected in both $J$ and $K$ bands could be shown; this is the reason why the number of stars is somewhat smaller than in Fig. 2. Finally, Fig. 4 shows the number of stars in our fields as a function of (the absolute value of the) galactic latitude $|b|$. It can be seen that the probability for a large number of detected stars in the field dramatically increases for smaller galactic latitudes. Our sample is too small to give a breakdown of the probability of finding reference stars at certain galactic latitudes, but it seems that the detected number of stars is in general somewhat smaller than predicted based on star counts in Reffert et al. ${ }^{5}$. However, our results are in excellent agreement with Creech-Eakman et al. ${ }^{13}$, who observed target fields in the northern hemisphere with a very similar purpose as described here. Extrapolating the results of Creech-Eakman et al., the probability of finding a reference star brighter than 16 mag in $K$ and closer than $10^{\prime \prime}$ to the target would be $12.3 \%$. In our survey, 12 probably unrelated field stars were found brighter than $K=16$ mag within $10^{\prime \prime}$ in 104 fields, yielding an average probability of $11.5 \%$. Another 16 physical pairs with angular separations smaller than $10^{\prime \prime}$ are present in the sample, so that in total $27 \%$ of our 104 fields would have suitable reference stars for the highest-precision measurements with PRIMA. Increasing the angular separation to 30 ", $66 \%$ of our 


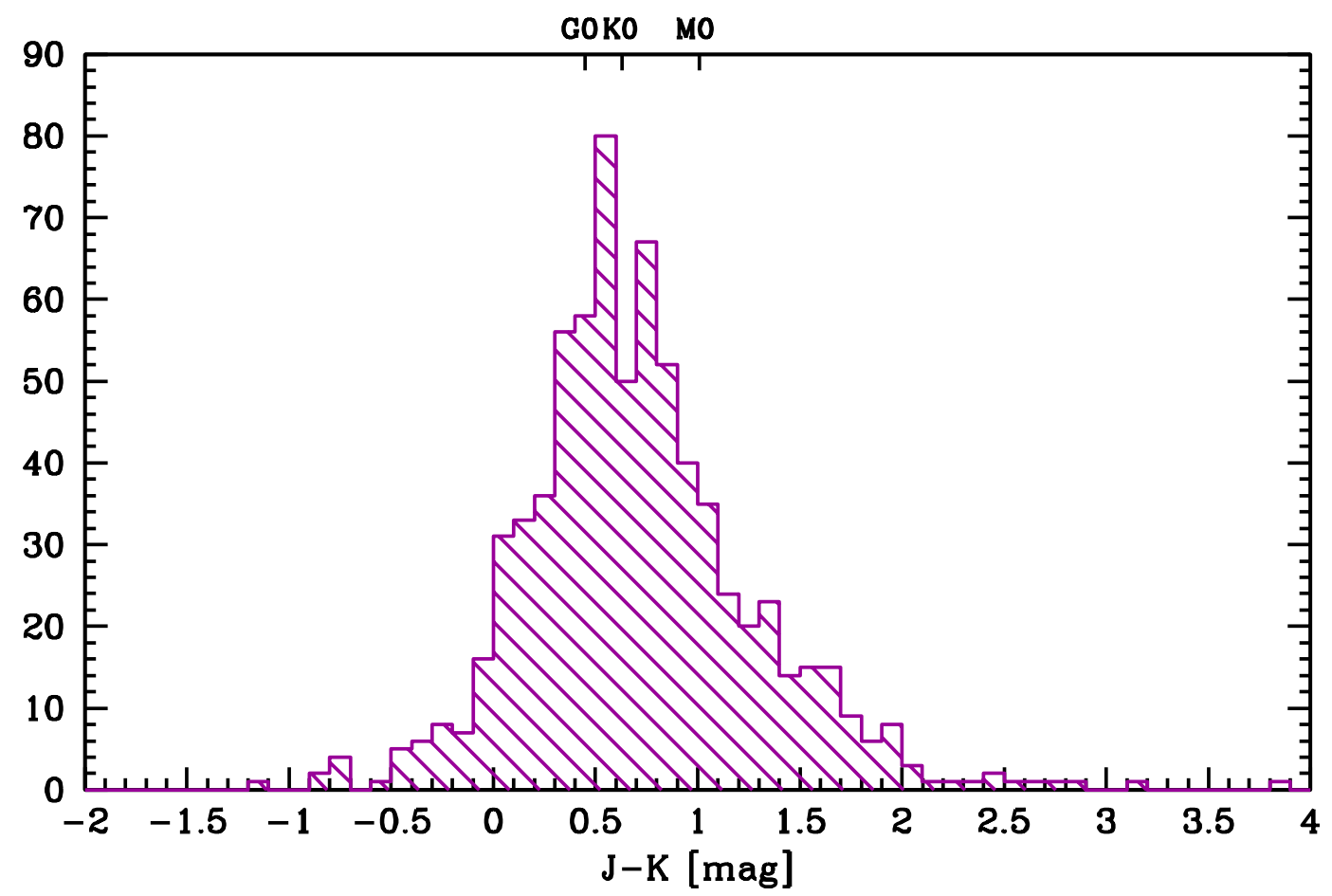

Figure 3. All 736 companions which have been detected in the $J$ and $K$ bands within $60^{\prime \prime}$ around the target star in 100 fields, including binary companions of comparable brightness as the target star. Most stars are of late spectral types, presumably unrelated field stars. The spectral types indicated on the top of the plot are as given by Bessell \& Brett ${ }^{12}$ assuming the stars are giants.

target stars would have possible reference stars brighter than $K=16$, including both physical companions as well as unrelated field stars.

These numbers look very favorable. However, there is still the possibility that the limit in $K$ magnitude currently considered will shift towards brighter magnitudes, which would diminish the numbers of fields observable with PRIMA. In any case, a larger number of fields has to be observed looking for possible reference stars than finally can be observed with PRIMA. In addition to the infrared imaging described here to identify possible reference stars, the target stars will be observed spectroscopically to identify unknown spectroscopic binaries among them; these cannot be easily used for our purposes. Also, this helps to better characterize our target stars, which is advantageous in the course of the astrometric data reduction. For the young star group, this will also enable an independent age estimate, so that we can be sure that the stars in this group are really young and active.

\section{COMPARISON WITH OTHER ASTROMETRIC PROJECTS}

Several other projects aim at detecting planets astrometrically. Benedict et al. ${ }^{14}$ succeeded already in detecting the astrometric signature of Gliese $876 \mathrm{~b}$ astrometrically using HST after it had been discovered with the radial velocity method. However, the method is rather observing time intensive, so that one can only look at a small number of targets, and the astrometric accuracy of HST is barely better than Hipparcos, so that not many more astrometric planet detections are expected from HST.

Another ground-based effort at Palomar is the STEPS survey (Pravdo et al. 2005), which currently observes about $30 \mathrm{M}$ dwarfs and also reaches accuracies similar to Hipparcos and HST. In principle, giant planets could be detected in this program, although none has been found yet. Again, the number of objects surveyed is relatively small, and the astrometric accuracy is just good enough to detect giant planets. 


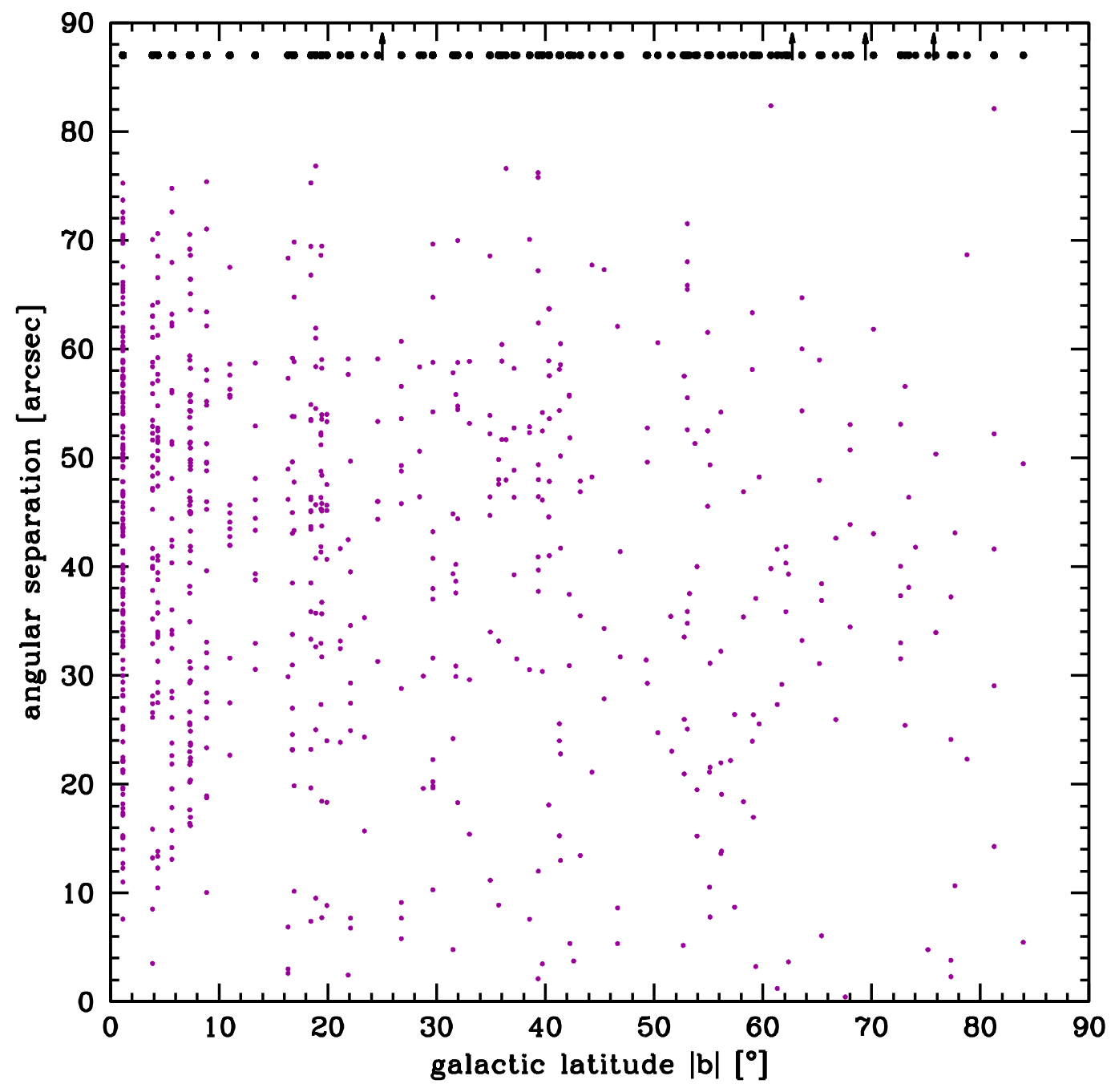

Figure 4. All 732 detections within about $80^{\prime \prime}$ of the target in each field, including binary companions of comparable brightness as the target star, as a function of the absolute value of the galactic latitude $|b|$. Not surprisingly, it can be seen how much more likely it is for a target at low galactic latitude to have several suitable reference stars for PRIMA (within about $30^{\prime \prime}$ of the target) than for targets at higher galactic latitudes. Four stars in our sample did not have any other stars detected in their fields; they are marked with arrows in the top row which also shows the distribution of galactic latitudes in our sample (one dot for each target star). 
This situation is expected to change dramatically with SIM Planetquest* (until recently scheduled for launch in 2011, but a delay of 4 years is possible) and $\mathrm{GAIA}^{\dagger}$, still scheduled for launch in 2011. Both will achieve a dramatic improvement in astrometric precision over Hipparcos. For SIM Planetquest the search for planets is the most important scientific goal. It is a targeted mission, so it will most probably observe a good number of the most nearby stars for planets as small as a few Earth masses. GAIA will scan the entire sky with unprecedented accuracy and depth, and it is estimated that it will detect thousands of giant planets.

The extrasolar planet search with PRIMA is unique among these projects since it will achieve a large jump in astrometric precision several years before the other two missions (SIM Planetquest and GAIA), and it is the only one which does it from the ground which makes it more flexible to adapt observing lists and plans. The number of targets which is observable is of the order of a few hundred, which holds the promise of some new exiting results. With a sensitivity down to Neptune mass planets around the most nearby stars it has the potential of detecting smaller mass planets around nearby $\mathrm{M}$ dwarfs than is possible with the radial velocity method, the existence of which is currently debated. Also, some synergies between PRIMA and SIM Planetquest could be expected, since PRIMA could provide promising stars to the SIM Planetquest target list.

\section{ACKNOWLEDGMENTS}

We kindly thank Hartmut Jahreiß for providing an updated version of the Catalog of Nearby Stars.

\section{REFERENCES}

1. A. Quirrenbach, T. Henning, D. Queloz, et al., "The PRIMA Astrometric Planet Search Project", Proc. SPIE 5491, 424, 2004

2. A. Quirrenbach, F. Delplancke, T.F.E. Henning, et al., "Calibration strategy and error budget for dual-star interferometry", Proc. SPIE 6268, 2006 (this volume)

3. F.A. Pepe, T.F.E. Henning, D. Queloz, A. Quirrenbach, et al., "Astrometric exoplanets search with PRIMA at the VLTI: the project", Proc. SPIE 6268, 2006 (this volume)

4. S. Frink, S. Hekker, R. Launhardt, J. Setiawan, D. Ségransan, A. Quirrenbach, T. Henning, D. Queloz, Proc. SPIE 5491, 1166, 2004

5. S. Reffert, R. Launhardt, S. Hekker, T. Henning, D. Queloz, A. Quirrenbach, D. Ségransan, J. Setiawan, "Choosing Suitable Target, Reference and Calibration Stars for the PRIMA Astrometric Planet Search", ASP Conf. Series 308, 81, 2005

6. A. Quirrenbach, "Interferometric high-resolution spectroscopy", Proc. SPIE 5491, 146, 2004

7. G. Marcy, R.P. Butler, D. Fischer, J.T. Wright, C.G. Tinney, H.R.A. Jones, "Observed Properties of Exoplanets: Masses, Orbits, and Metallicities", Progress of Theoretical Physics Supplement 158, 24, 2005

8. M. Shao, M.M. Colavita, "Potential of long-baseline infrared interferometry for narrow-angle astrometry", A $\& A$ 262, 353, 1992

9. O. von der Lühe, A. Quirrenbach, B. Koehler, "Narrow-Angle Astrometry with the VLT Interferometer", in: Science with the VLT, eds. J.R.Walsh \& I.J.Danziger, Springer-Verlag, 445, 1995

10. M.W. Muterspaugh, B.F. Lane, M. Konacki, B.F. Burke, M.M. Colavita, S.R. Kulkarni, M. Shao, "PHASES High-Precision Differential Astrometry of $\delta$ Equulei", $A J$ 130, 2866, 2005

11. Hartkopf, W.I., Mason, B.D., "Sixth Catalog of Orbits of Visual Binary Stars", 2005, available at http://ad.usno.navy.mil/wds/orb6.html

12. M. S. Bessell, J. M. Brett, "JHKLM photometry - Standard systems, passbands, and intrinsic colors", PASP 100, 1134,1988

13. M.J. Creech-Eakman, S.R. Kulkarni, X.P. Pan, S.B. Shaklan, "Photometric Measurements of the Fields of More than 700 Nearby Stars", AJ 118, 2483, 1999

14. G.F. Benedict et al., "A Mass for the Extrasolar Planet Gliese 876b Determined from Hubble Space Telescope Fine Guidance Sensor 3 Astrometry and High-Precision Radial Velocities", APJ 581, L115, 2002

15. S.H. Pravdo, S.B. Shaklan, J. Lloyd, J., G.F. Benedict, "Discovering M-Dwarf Companions with STEPS", ASP Conf. Series 338, 288, 2005

\footnotetext{
${ }^{*}$ http://sim.jpl.nasa.gov/
}

${ }^{\dagger}$ http://gaia.esa.int/ 